\title{
Period Matrices of Polyhedral Surfaces
}

\author{
Alexander Bobenko*1, Christian Mercat ${ }^{2}$, and Markus Schmies ${ }^{1}$ \\ 1 Technische Universität Berlin, Institut für Mathematik, Strasse des 17. Juni 136, \\ 10623 Berlin, Germany, bobenko, schmies@math.tu-berlin.de \\ 2 I3M c.c. 51, Université Montpellier 2, F-34095 Montpellier cedex 5, France \\ mercat@math.univ-montp2.fr
}

\section{Introduction}

Finding a conformal parameterization for a surface and computing its period matrix is a classical problem which is useful in a lot of contexts, from statistical mechanics to computer graphics.

The 2D-Ising model [Mer01, CSM02, CSM03] for example can be realized on a cellular decomposition of a surface whose edges are decorated by interaction constants, understood as a discrete conformal structure. In certain configurations, called critical temperature, the model exhibits conformal invariance properties in the thermodynamical limit and certain statistical expectations become discrete holomorphic at the finite level. The computation of the period matrix of higher genus surfaces built from the rectangular and triangular lattices from discrete Riemann theory has been addressed in the cited papers by Costa-Santos and McCoy.

Global conformal parameterization of a surface is important in computer graphics [JWYG04, DMA02, BCGB08, TACSD06, KSS06, War06, SSP08] in issues such as texture mapping of a flat picture onto a curved surface in $\mathbb{R}^{3}$. When the texture is recognized by the user as a natural texture known as featuring round grains, these features should be preserved when mapped onto the surface, mainly because any shear of circles into ellipses is going to be wrongly interpreted as suggesting depth increase. Characterizing a surface by a few numbers is as well a desired feature in computer graphics, for problems like pattern recognition. Computing numerically the period matrix of a surface has been addressed in [JWYG04].

This chapter uses the general framework of the theory of discrete Riemann surfaces [Fer44, Duf68, Mer01, BMS05] and the computation of period matrices within this framework (based on theorems and not only numerical analogies). Other approaches have emerged recently [GN07, DN03, Kis08].

* This work is supported by the DFG Research Unit Polyhedral Surfaces. 
We focus here on the computation of period matrices within this framework (based on theorems and not only numerical analogies).

We start with some surfaces with known period matrices and compute numerically their discrete period matrices, at different levels of refinement. In particular, some genus two surfaces made up of squares and the Wente torus are considered. We observe numerically good convergence properties. Moreover, we compute the yet unknown period matrix of the Lawson surface, identify it numerically as one of the tested surfaces, which allows us to conjecture their conformal equivalence, and finally to prove it.

\section{Discrete conformal structure}

Consider a polyhedral surface in $\mathbb{R}^{3}$. It has a unique Delaunay tessellation, generically a triangulation [BS07]. That is to say each face is associated with a circumcircle drawn on the surface and this disk contains no other vertices than the ones on its boundary. Let's call $\Gamma$ the graph of this cellular decomposition, $\Gamma_{0}$ its vertices, $\Gamma_{1}$ its edges and complete it into a cellular decomposition with $\Gamma_{2}$ the set of triangles. Each edge $\left(x, x^{\prime}\right)=e \in \Gamma_{1}$ is adjacent to a pair of triangles, associated with two circumcenters $y, y^{\prime}$. The ratio of the (intrinsic) distances between the circumcenters and the length of the (orthogonal) edge $e$ is called $\rho(e)$. It is the celebrated cotan formula [PP93].

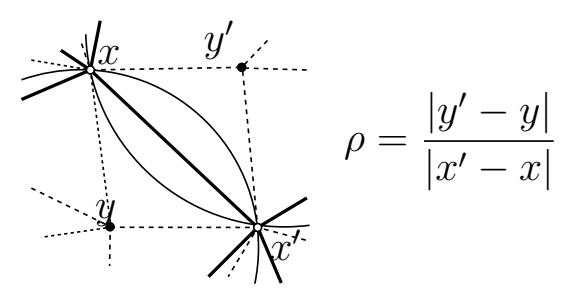

Fig. 1. The ratio of the intrinsic lengths of the diagonals is the cotan weight

Following [Mer01], we call these data of a graph $\Gamma$, whose edges are equipped with a positive real number, a discrete conformal structure. A discrete Riemann surface is a conformal equivalence class of surfaces with the same discrete conformal structure. It leads to a theory of discrete Riemann surfaces and discrete analytic functions, developed in [Fer44, Duf68, Mer01, Mer04, Mer07, BMS05, DKT08], that shares a lot of features with the continuous theory, and these features are recovered in a proper refinement limit. We are going to summarize these results.

In our examples, the extrinsic triangulations are Delaunay. That is to say the triangulations come from the embedding in $\mathbb{R}^{3}$ and the edges $\left(x, x^{\prime}\right) \in \Gamma_{1}$ of the triangulation are the edges of the polyhedral surface in $\mathbb{R}^{3}$. On the other hand, the geodesic connecting the circumcenters $y$ and $y^{\prime}$ on the surface 
is not an interval of a straight line and its length is generically greater than the distance $\left\|y-y^{\prime}\right\|$ in $\mathbb{R}^{3}$. The latter gives a more naive definition of $\rho$ and is easier to compute. We will call it extrinsic, because in contrast to the intrinsic $\rho$, it depends on the embedding of the surface in $\mathbb{R}^{3}$ and is not preserved by isometries. For surfaces which are refined and flat enough, the difference between extrinsic and intrinsic distances is not large. We compare numerically the two ways to compute $\rho$. The conclusion is that, in the examples we tested, the intrinsic distance is marginally better, see Sect. 4.2.

The circumcenters and their adjacencies define a 3 -valent abstract (locally planar) graph, dual to the graph of the surface, that we call $\Gamma^{*}$, with vertices $\Gamma_{0}^{*}=\Gamma_{2}$, edges $\Gamma_{1}^{*} \simeq \Gamma_{1}$. We equip the edge $\left(y, y^{\prime}\right)=e^{*} \in \Gamma_{1}^{*}$, dual to the primal edge $e \in \Gamma_{1}$, with the positive real constant $\rho\left(e^{*}\right)=1 / \rho(e)$. We define $\Lambda:=\Gamma \oplus \Gamma^{*}$ the double graph, with vertices $\Lambda_{0}=\Gamma_{0} \sqcup \Gamma_{0}^{*}$ and edges $\Lambda_{1}=$ $\Gamma_{1} \sqcup \Gamma_{1}^{*}$. Each pair of dual edges $e, e^{*} \in \Lambda_{1}, e=\left(x, x^{\prime}\right) \in \Gamma_{1}, e^{*}=\left(y, y^{\prime}\right) \in \Gamma_{1}^{*}$, is seen as the diagonals of a quadrilateral $\left(x, y, x^{\prime}, y^{\prime}\right)$, composing a quad-graph $\diamond$, with vertices $\nabla_{0}=\Lambda_{0}$, edges $\nabla_{1}$ composed of couples $(x, y)$ and faces $\nabla_{2}$ composed of quadrilaterals $\left(x, y, x^{\prime}, y^{\prime}\right)$.

The Hodge star, which in the continuous theory is defined by $*(f \mathrm{~d} x+$ $g \mathrm{~d} y)=-g \mathrm{~d} x+f \mathrm{~d} y$, is in the discrete case the duality transformation multiplied by the conformal structure:

$$
\int_{e^{*}} * \alpha:=\rho(e) \int_{e} \alpha
$$

A 1-form $\alpha \in C^{1}(\Lambda)$ is of type $(1,0)$ if and only if, for each quadrilateral $\left(x, y, x^{\prime}, y^{\prime}\right) \in \diamond_{2}, \int_{\left(y, y^{\prime}\right)} \alpha=\mathrm{i} \rho\left(x, x^{\prime}\right) \int_{\left(x, x^{\prime}\right)} \alpha$, that is to say if $* \alpha=-\mathrm{i} \alpha$. Similarly forms of type $(0,1)$ are defined by $* \alpha=+\mathrm{i} \alpha$. A form is holomorphic, respectively anti-holomorphic, if it is closed and of type $(1,0)$, respectively of type $(0,1)$. A function $f: \Lambda_{0} \rightarrow \mathbb{C}$ is holomorphic iff $\mathrm{d}_{\Lambda} f$ is.

We define a wedge product for 1 -forms living either on edges $\rangle_{1}$ or on their diagonals $\Lambda_{1}$, as a 2 -form living on faces $\diamond_{2}$. The formula for the latter is:

$$
\iint_{\left(x, y, x^{\prime}, y^{\prime}\right)} \alpha \wedge \beta:=\frac{1}{2}\left(\int_{\left(x, x^{\prime}\right)} \alpha \int_{\left(y, y^{\prime}\right)} \beta-\int_{\left(y, y^{\prime}\right)} \alpha \int_{\left(x, x^{\prime}\right)} \beta\right)
$$

The exterior derivative $\mathrm{d}$ is a derivation for the wedge product, for functions $f, g$ and a 1-form $\alpha$ :

$$
\mathrm{d}(f g)=f \mathrm{~d} g+g \mathrm{~d} f, \quad \mathrm{~d}(f \alpha)=\mathrm{d} f \wedge \alpha+f \mathrm{~d} \alpha .
$$

Together with the Hodge star, they give rise, in the compact case, to the usual scalar product on 1-forms:

$$
(\alpha, \beta):=\iint_{\diamond_{2}} \alpha \wedge * \bar{\beta}=(* \alpha, * \beta)=\overline{(\beta, \alpha)}=\frac{1}{2} \sum_{e \in \Lambda_{1}} \rho(e) \int_{e} \alpha \int_{e} \bar{\beta}
$$


The adjoint $\mathrm{d}^{*}=-* \mathrm{~d} *$ of the coboundary $\mathrm{d}$ allows to define the discrete Laplacian $\Delta=\mathrm{d}^{*} \mathrm{~d}+\mathrm{d} \mathrm{d}^{*}$, whose kernel are the harmonic forms and functions. It reads, for a function at a vertex $x \in \Lambda_{0}$ with neighbours $x^{\prime} \sim x$ :

$$
(\Delta f)(x)=\sum_{x^{\prime} \sim x} \rho\left(x, x^{\prime}\right)\left(f(x)-f\left(x^{\prime}\right)\right) .
$$

Hodge theorem: The two \pm i-eigenspaces decompose the space of 1-forms, especially the space of harmonic forms, into an orthogonal direct sum. Types are interchanged by conjugation: $\alpha \in C^{(1,0)}(\Lambda) \Leftrightarrow \bar{\alpha} \in C^{(0,1)}(\Lambda)$ therefore

$$
(\alpha, \beta)=\left(\pi_{(1,0)} \alpha, \pi_{(1,0)} \beta\right)+\left(\pi_{(0,1)} \alpha, \pi_{(0,1)} \beta\right)
$$

where the projections on $(1,0)$ and $(0,1)$ spaces are

$$
\pi_{(1,0)}=\frac{1}{2}(\mathrm{Id}+\mathrm{i} *), \quad \pi_{(0,1)}=\frac{1}{2}(\mathrm{Id}-\mathrm{i} *) .
$$

The harmonic forms of type $(1,0)$ are the holomorphic forms, the harmonic forms of type $(0,1)$ are the anti-holomorphic forms.

The $L^{2}$ norm of the 1 -form $\mathrm{d} f$, called the Dirichlet energy of the function $f$, is the average of the usual Dirichlet energies on each independent graph

$$
\begin{aligned}
E_{D}(f):=\|\mathrm{d} f\|^{2} & =(\mathrm{d} f, \mathrm{~d} f)=\frac{1}{2} \sum_{\left(x, x^{\prime}\right) \in \Lambda_{1}} \rho\left(x, x^{\prime}\right)\left|f\left(x^{\prime}\right)-f(x)\right|^{2} \\
& =\frac{E_{D}\left(\left.f\right|_{\Gamma}\right)+E_{D}\left(\left.f\right|_{\Gamma^{*}}\right)}{2} .
\end{aligned}
$$

The conformal energy of a map measures its conformality defect, relating these two harmonic functions. A conformal map fulfills the Cauchy-Riemann equation

$$
* \mathrm{~d} f=-\mathrm{i} \mathrm{d} f .
$$

Therefore a quadratic energy whose null functions are the holomorphic ones is

$$
E_{C}(f):=\frac{1}{2}\|\mathrm{~d} f-\mathrm{i} * \mathrm{~d} f\|^{2} .
$$

It is related to the Dirichlet energy through the same formula as in the continuous case:

$$
\begin{aligned}
E_{C}(f) & =\frac{1}{2}(\mathrm{~d} f-\mathrm{i} * \mathrm{~d} f, \mathrm{~d} f-\mathrm{i} * \mathrm{~d} f) \\
& =\frac{1}{2}\|\mathrm{~d} f\|^{2}+\frac{1}{2}\|-\mathrm{i} * \mathrm{~d} f\|^{2}+\operatorname{Re}(\mathrm{d} f,-\mathrm{i} * \mathrm{~d} f) \\
& =\|\mathrm{d} f\|^{2}+\operatorname{Im} \iint_{\diamond_{2}} \mathrm{~d} f \wedge \overline{d f} \\
& =E_{D}(f)-2 \mathcal{A}(f)
\end{aligned}
$$

where the area of the image of the map $f$ in the complex plane has the same formulae (the second one meaningful on a simply connected domain) 


$$
\mathcal{A}(f)=\frac{\mathrm{i}}{2} \iint_{\diamond_{2}} \mathrm{~d} f \wedge \overline{\mathrm{d} f}=\frac{\mathrm{i}}{4} \oint_{\partial \diamond_{2}} f \overline{\mathrm{d} f}-\bar{f} \mathrm{~d} f
$$

as in the continuous case. For a face $\left(x, y, x^{\prime}, y^{\prime}\right) \in \diamond_{2}$, the algebraic area of the oriented quadrilateral $\left(f(x), f\left(x^{\prime}\right), f(y), f\left(y^{\prime}\right)\right)$ is given by

$$
\begin{aligned}
\iint_{\left(x, y, x^{\prime}, y^{\prime}\right)} \mathrm{d} f \wedge \overline{\mathrm{d} f} & =\mathrm{i} \operatorname{Im}\left(\left(f\left(x^{\prime}\right)-f(x)\right) \overline{\left(f\left(y^{\prime}\right)-f(y)\right)}\right) \\
& =-2 \mathrm{i} \mathcal{A}\left(f(x), f\left(x^{\prime}\right), f(y), f\left(y^{\prime}\right)\right) .
\end{aligned}
$$

When a holomorphic reference map $z: \Lambda_{0} \rightarrow \mathbb{C}$ is chosen, a holomorphic (resp. anti-holomorphic) 1-form $\mathrm{d} f$ is, locally on each pair of dual diagonals, proportional to $\mathrm{d} z$, resp. $\mathrm{d} \bar{z}$, so that the decomposition of the exterior derivative into holomorphic and anti-holomorphic parts yields $\mathrm{d} f \wedge \overline{\mathrm{d} f}=$ $\left(|\partial f|^{2}+|\bar{\partial} f|^{2}\right) \mathrm{d} z \wedge \mathrm{d} \bar{z}$ where the derivatives naturally live on faces and are not to be mixed up with the boundary operator $\partial$.

\section{Algorithm}

The theory described above is straightforward to implement. The most sensitive part is based on a minimizer procedure which finds the minimum of the Dirichlet energy for a discrete Riemann surface, given some boundary conditions. Here is the crude algorithm that we are going to detail.

The intersection number $\gamma \circ \gamma^{\prime}$ between two loops $\gamma$ and $\gamma^{\prime}$ on a surface counts their algebraic number of crossings. It is a homotopy invariant and has a clear discrete counterpart.

A canonical dissection $\aleph$ is a normalized homotopy basis of a quad-graph $\diamond(S)$, that is to say, a set $\left(\aleph_{k}\right)_{1 \leq k \leq 2 g}$ of loops $\aleph_{k} \in \operatorname{ker} \partial$ such that the intersection numbers verify, for $1 \leq k, \ell \leq g$,

$$
\aleph_{k} \circ \aleph_{\ell}=0, \quad \aleph_{k+g} \circ \aleph_{\ell+g}=0, \quad \aleph_{k} \circ \aleph_{\ell+g}=\delta_{k, \ell} .
$$

The situation is again doubled and a loop $\aleph_{k}$ is pushed on the graph $\Gamma$ to the cycle $\aleph_{k}^{\Gamma}$ and on its dual $\Gamma^{*}$ to $\aleph_{k}^{\Gamma^{*}}$. See [Mer07] for more details.

Basis of holomorphic forms and the period matrix (of a discrete Riemann surface $S$ )

- find a normalized (9) homotopy basis $\aleph$ of $\diamond(S)$ and compute $\aleph_{k}^{\Gamma}$ and $\aleph_{k}^{\Gamma^{*}}$,

- compute the real discrete harmonic forms $\omega_{k}$ on $\Gamma$ such that $\oint_{\gamma} \omega_{k}=\gamma \circ \aleph_{k}^{\Gamma}$,

- compute the dual discrete forms $* \omega_{k}$ on $\Gamma^{*}$, check $* \omega_{k}$ is discrete harmonic on $\Gamma^{*}$,

- compute its periods $\left(\oint_{\aleph_{\ell}^{\Gamma^{*}}} * \omega_{k}\right)_{k, \ell}$ on $\Gamma^{*}$,

- do likewise for the analogous discrete harmonic forms $\omega_{k}^{*}$ on $\Gamma^{*}$, 
- find the basis of holomorphic forms $\left(\zeta_{k}\right)$ normalized by

$$
\oint_{\aleph_{\ell}^{\Gamma}} \zeta_{k}=\delta_{k, \ell}, \oint_{\aleph_{\ell}^{\Gamma^{*}}} \zeta_{k}=0,1 \leq k, \ell \leq g .
$$

This can be done by some linear algebra $(\zeta)=R(\mathrm{Id}+\mathrm{i} *)(\omega)(R$ is a rectangular complex matrix).

- define the period matrix of $\Gamma, \Pi_{k, \ell}:=\oint_{\aleph_{g+\ell}^{*}} \zeta_{k}+\oint_{\aleph_{g+\ell}} \zeta_{k}$ for $1 \leq \ell, k \leq g$,

- do likewise for $\zeta_{k}^{*}$ normalized by

$$
\oint_{\aleph_{\ell}^{\Gamma^{*}}} \zeta_{k}^{*}=\delta_{k, \ell}, \oint_{\aleph_{\ell}^{\Gamma}} \zeta_{k}^{*}=0,1 \leq k, \ell \leq g,
$$

- define the period matrix of $\Gamma, \Pi_{k, \ell}^{*}:=\oint_{\aleph_{g+\ell}} \zeta_{k}^{*}+\oint_{\aleph_{g+\ell}^{*}} \zeta_{k}^{*}$,

- define the period matrix as the half-sum

$$
\frac{1}{2}\left(\Pi+\Pi^{*}\right) .
$$

Finding a normalized homotopy basis of a connected cellular decomposition is performed by several well known algorithms. The way we did it is to select a root vertex and grow from there a spanning tree, by computing the vertices at combinatorial distance $d$ from the root and linking each one of them to a unique vertex at distance $d-1$, already in the tree. Repeat until no vertices are left.

Then we inflate this tree into a polygonal fundamental domain by adding faces one by one to the domain, keeping it simply connected: We recursively add all the faces which have only one edge not in the domain. We stop when all the remaining faces have at least two edges not in the domain (see Fig. 2).

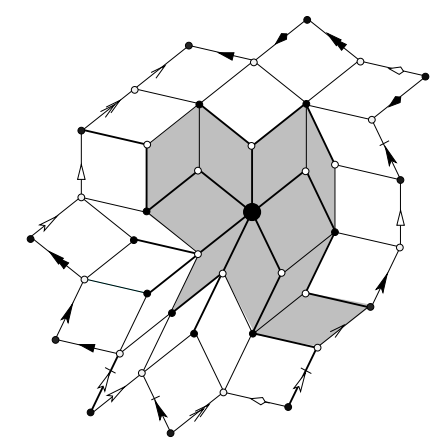

Fig. 2. A maximal spanning tree and its first inflation step.

Then we pick one edge (one of the closest to the root) as defining the first element of our homotopy basis: adding this edge to the fundamental domain 
yields a non simply connected cellular decomposition and the spanning tree gives us a rooted cycle of this homotopy type going down the tree to the root. It is (one of) the combinatorially shortest in its (rooted) homology class. We add faces recursively in a similar way until we can no go further. Then we choose a new homotopy basis element, and so on until every face is closed (see Fig. 3). At the end we have a homotopy basis. We compute later on the intersection numbers in order to normalize the basis.
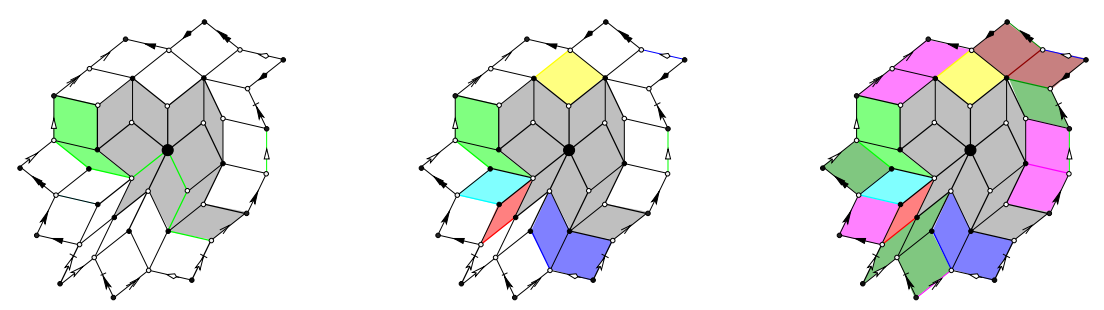

Fig. 3. A homotopy class is associated with an edge linking two sides of the polygon. Here a genus 4 example.

We compute the unique real harmonic form $\eta$ associated with each cycle $\aleph$ such that $\oint_{\gamma} \eta=\gamma \circ \aleph$. This is done by a minimizing procedure which finds the unique harmonic function $f$ on the graph $\Gamma$, split along $\aleph$, whose vertices are duplicated, which is zero at the root and increases by one when going across $\aleph$. This is done by linking the values at the duplicated vertices, in effect yielding a harmonic function on the universal cover of $\Gamma$. The harmonic 1 -form $\mathrm{d} f$ does not depend on the chosen root nor on the representative $\aleph$ in its homology class.

\section{Numerics}

We begun with testing discrete surfaces of known moduli in order to investigate the quality of the numerics and the robustness of the method. We purposely chose to stick with raw double 15 -digits numbers and a linear algebra library which is fast but not particularly accurate. In order to be able to compare period matrices, we used a Siegel reduction algorithm $\left[\mathrm{DHB}^{+} 04\right]$ to map them by a modular transformation to the same canonical form.

\subsection{Surfaces tiled by squares}

Robert Silhol supplied us with sets of surfaces tiled by squares for which the period matrices are known [Sil06, Sil01, BS01, RGA97]. There are translation and half-translation surfaces: In these surfaces, each horizontal side is glued to a horizontal side, a vertical to a vertical, and the identification between 
edges of the fundamental polygon are translations for translation surfaces and translations followed by a half-twist for half-translations. The discrete conformal structure for these surfaces is very simple: the combinatorics is given by the gluing conditions and the conformal parameter $\rho \equiv 1$ is constant.

The genus one examples are not interesting because this 1-form is then the unique holomorphic form and there is nothing to compute (the algorithm does give back this known result). Genus 2 examples are non trivial because a second holomorphic form has to be computed.

The translation surfaces are particularly adapted because the discrete 1form read off the picture is already a discrete holomorphic form. Therefore the computations are accurate even for a small number of squares. Finer squares only blur the result with numerical noise. For half-translation surfaces this is not the case, a continuous limit has to be taken in order to get a better approximation.

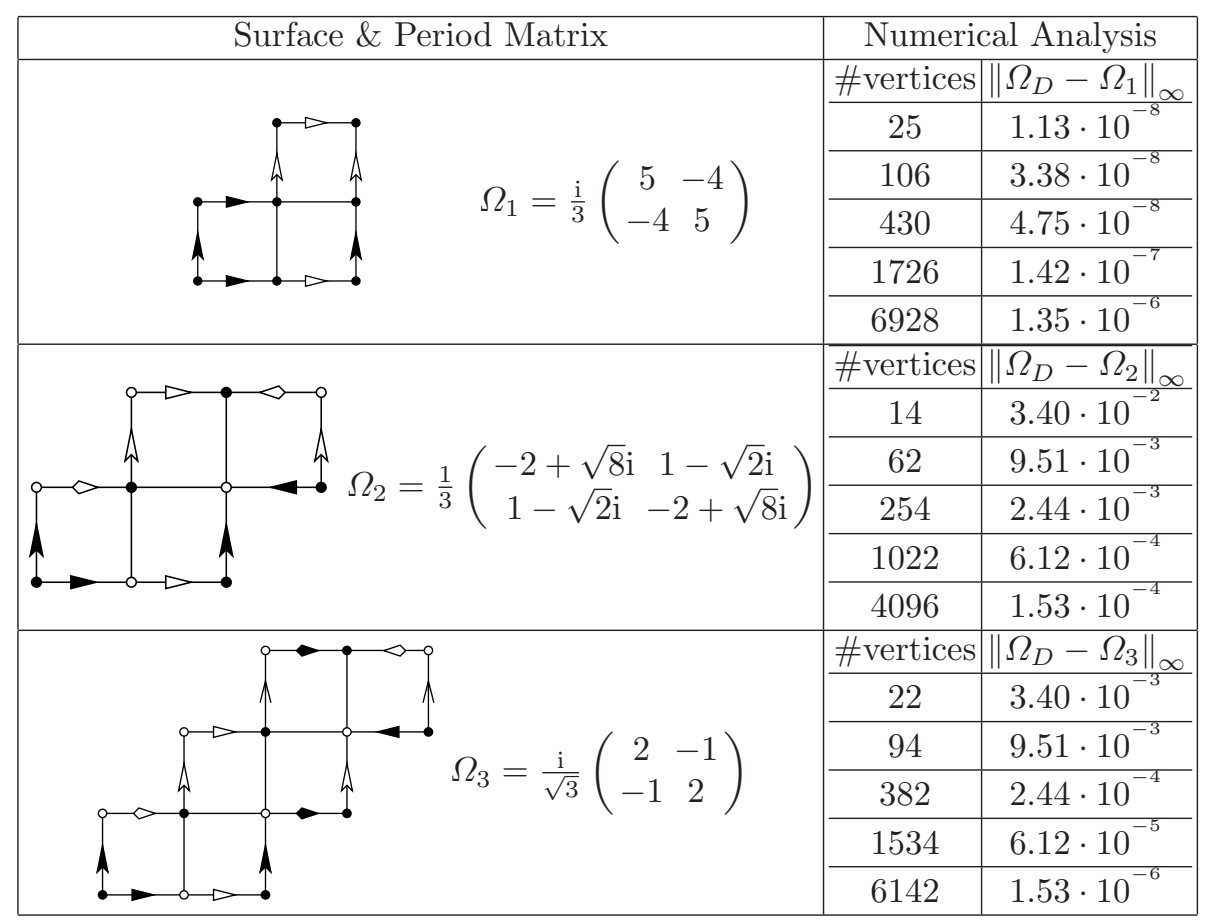

Using 15 digits numbers, the theoretical numerical accuracy is limited to 8 digits because our energy is quadratic therefore half of the digits are lost. Using an arbitrary precision toolbox or Cholesky decomposition in order to solve the linear system would allow for better results but this is not the point here. 

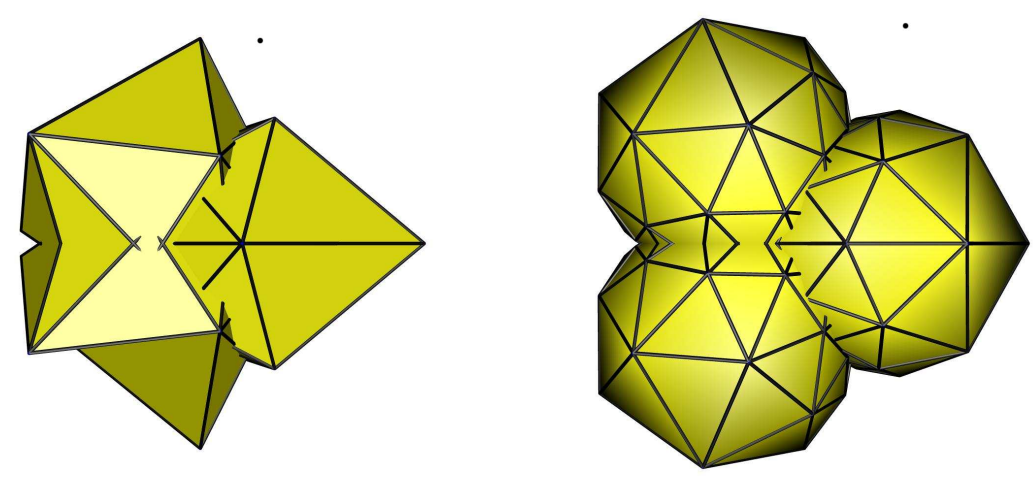

Grid : $10 \times 10$

Grid : $20 \times 20$
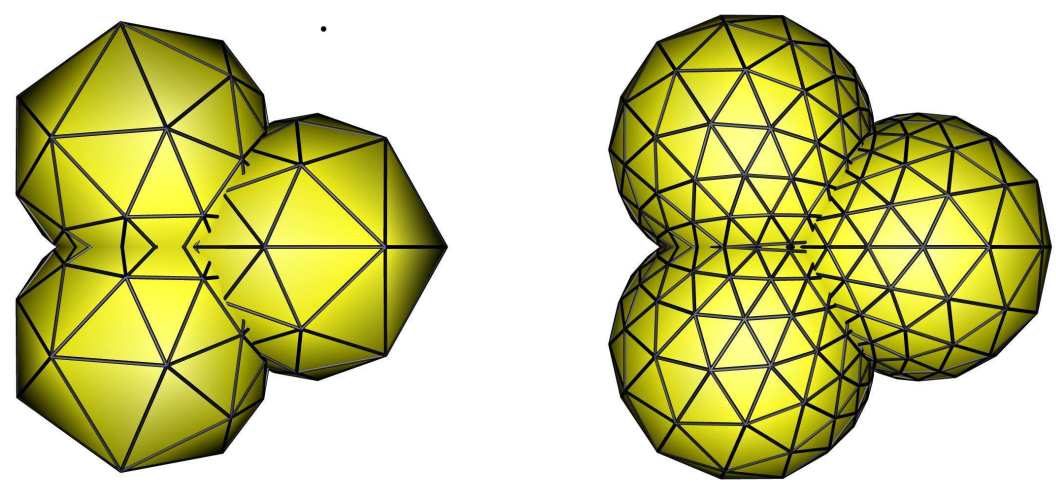

Grid : $40 \times 40$

Grid : $80 \times 80$

Fig. 4. Regular Delaunay triangulations of the Wente torus

\subsection{Wente torus}

For a first test of the numerics on a an immersed surface in ${ }^{3}$ our choice is the famous CMC-torus discovered by Wente [Wen86] for which an explicit immersion formula exists in terms of theta functions [Abr87, Wal89, Bob91]. The modulus of the rhombic Wente torus is known [Hei96] to be equal to:

$$
\tau_{w} \approx 0.41300 \ldots+0.91073 \ldots \mathrm{i} \approx \exp (\mathrm{i} 1.145045 \ldots) .
$$

We compute several regular discretizations of the Wente torus (Fig. 4) and generate discrete conformal structures using $\rho_{\text {ex }}$ that are imposed by the extrinsic Euclidean metric of ${ }^{3}$ as well as $\rho_{\text {in }}$ which are given by the intrinsic 
flat metric of the surface. For a sequence of finer discretizations of a smooth immersion, the two sets of numbers come closer and closer. For these discrete conformal structures we compute again the moduli which we denote by $\tau_{\text {ex }}$ and $\tau_{\mathrm{im}}$ and compare them with $\tau_{w}$ from above:

\begin{tabular}{|c|l|l|}
\hline Grid & $\left\|\tau_{\text {in }}-\tau_{w}\right\|$ & $\left\|\tau_{\text {ex }}-\tau_{w}\right\|$ \\
\hline $10 \times 10$ & $5.69 \cdot 10^{-3}$ & $5.00 \cdot 10^{-3}$ \\
\hline $20 \times 20$ & $2.00 \cdot 10^{-3}$ & $5.93 \cdot 10^{-3}$ \\
\hline $40 \times 40$ & $5.11 \cdot 10^{-4}$ & $1.85 \cdot 10^{-3}$ \\
\hline $80 \times 80$ & $2.41 \cdot 10^{-4}$ & $6.00 \cdot 10^{-4}$ \\
\hline
\end{tabular}

For the lowest resolution the accuracy of $\tau_{\text {ex }}$ is slightly better then the one of $\tau_{\text {in }}$. For all other the discrete conformal structures with the intrinsically generated $\rho_{\text {in }}$ yields significant higher accuracy.

\subsection{Lawson surface}

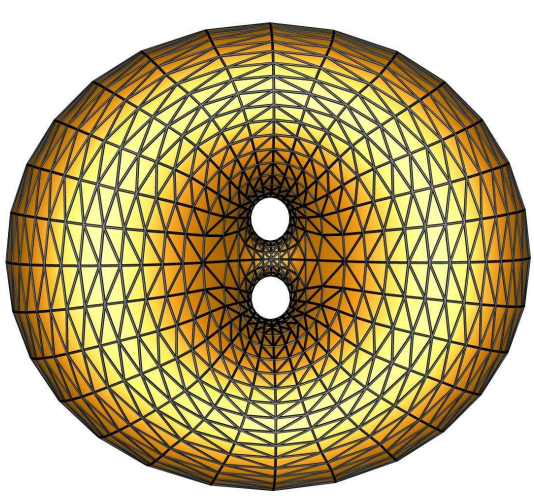

1162 vertices

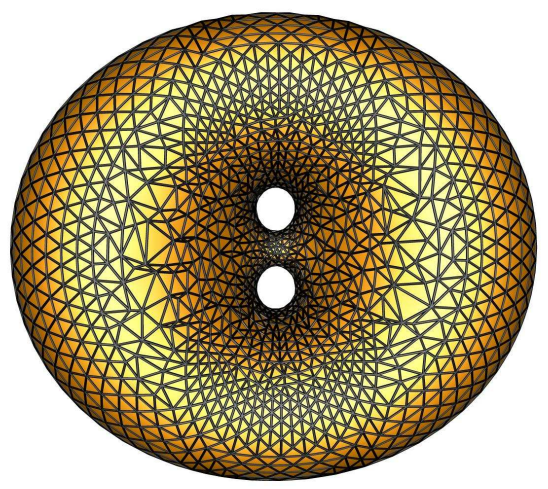

2498 vertices

Fig. 5. Delaunay triangulations of the Lawson surface

Finally we apply our method to compute the period matrix of Lawson's genus 2 minimal surface in $\mathbb{S}^{3}$ [Law70]. Konrad Polthier [GBP96] supplied us with several resolutions of the surface which are generated by a coarsening and mesh beautifying process of a very fine approximation of the Lawson surface (Fig. 5). Our numerical analysis gives evidence that the period matrix of the Lawson surface is

$$
\Omega_{l}=\frac{\mathrm{i}}{\sqrt{3}}\left(\begin{array}{cc}
2 & -1 \\
-1 & 2
\end{array}\right)
$$


which equals the period matrix $\Omega_{3}$ of the third example from Sect. 4.1. Once conjectured that these two surfaces are conformally equivalent, it is a matter of checking that the symmetry group of the Lawson genus two surface yields indeed this period matrix, which was done, without prior connection, in [BBM85]. An explicit conformal mapping of the surfaces can be found manually: The genus 2 Lawson surface exhibits by construction four points with an order six symmetry and six points of order four, which decomposes the surface into 24 conformally equivalent triangles, of angles $\frac{\pi}{6}, \frac{\pi}{2}, \frac{\pi}{2}$. Therefore an algebraic equation for the Lawson surface is $y^{2}=x^{6}-1$, with six branch points at the roots of unity. The correspondence between the points in the square picture of the surface and the double sheeted cover of the complex plane is done in Fig. 6. In particular the center of the six squares are sent to the branch points, the vertices are sent to the two copies of 0 (black and dark gray) and $\infty$ (white and light gray), the squares are sent to double sheeted 2 -gons corresponding to a sextant.

Similarly to Sect. 4.2 we compute the period matrices $\Omega_{\mathrm{ex}}$ and $\Omega_{\text {in }}$ for different resolutions utilizing weights imposed by the extrinsic and intrinsic metric and compare the results with our conjectured period matrix for the Lawson surface $\Omega_{l}$ :

\begin{tabular}{|c|c|c|}
\hline \#vertices & $\left\|\Omega_{\mathrm{in}}-\Omega_{l}\right\|_{\infty}$ & $\left\|\Omega_{\mathrm{ex}}-\Omega_{l}\right\|_{\infty}$ \\
\hline 1162 & $1.68 \cdot 10^{-3}$ & $1.68 \cdot 10^{-3}$ \\
\hline 2498 & $3.01 \cdot 10^{-3}$ & $3.20 \cdot 10^{-3}$ \\
\hline 10090 & $8.55 \cdot 10^{-3}$ & $8.56 \cdot 10^{-3}$ \\
\hline
\end{tabular}

Our first observation is that the matrices $\Omega_{\mathrm{ex}}$ and $\Omega_{\text {in }}$ almost coincide. Hence the method for computing the $\rho$ seems to have only little influence on this result (compare also Sect. 4.2). Further we see that figures of the higher resolution surface, i.e., with 2498 and 10090 vertices are worse than the coarsest one with 1162 vertices. The mesh beautifying process was most successful on the coarsest triangulation of the Lawson surface (Fig. 5). The quality of the mesh has a significant impact on the accuracy of our computation: One can see that the triangles on the coarsest example are of even shapes with comparable side lengths, while the finer resolution contains thin triangles with small angles. The convergence speed proven in [Mer01] and used in [Mer07] for period matrices is governed by this smallest angles, accounting for the poor result. Therefore for this method to be applicable, the data should be well suited. It is not enough to have very refined data if the triangles themselves are not of a good shape. A further impediment to the method is the fact that the triangulation should be Delaunay. If it is not, it can be repaired by the algorithm described in [FSSB07].

\section{Acknowledgment:}

This work is partially supported by the DFG Research Unit 'Polyhedral Surfaces' and by the DFG Research Center 'Matheon'. 
228 Alexander Bobenko, Christian Mercat, and Markus Schmies
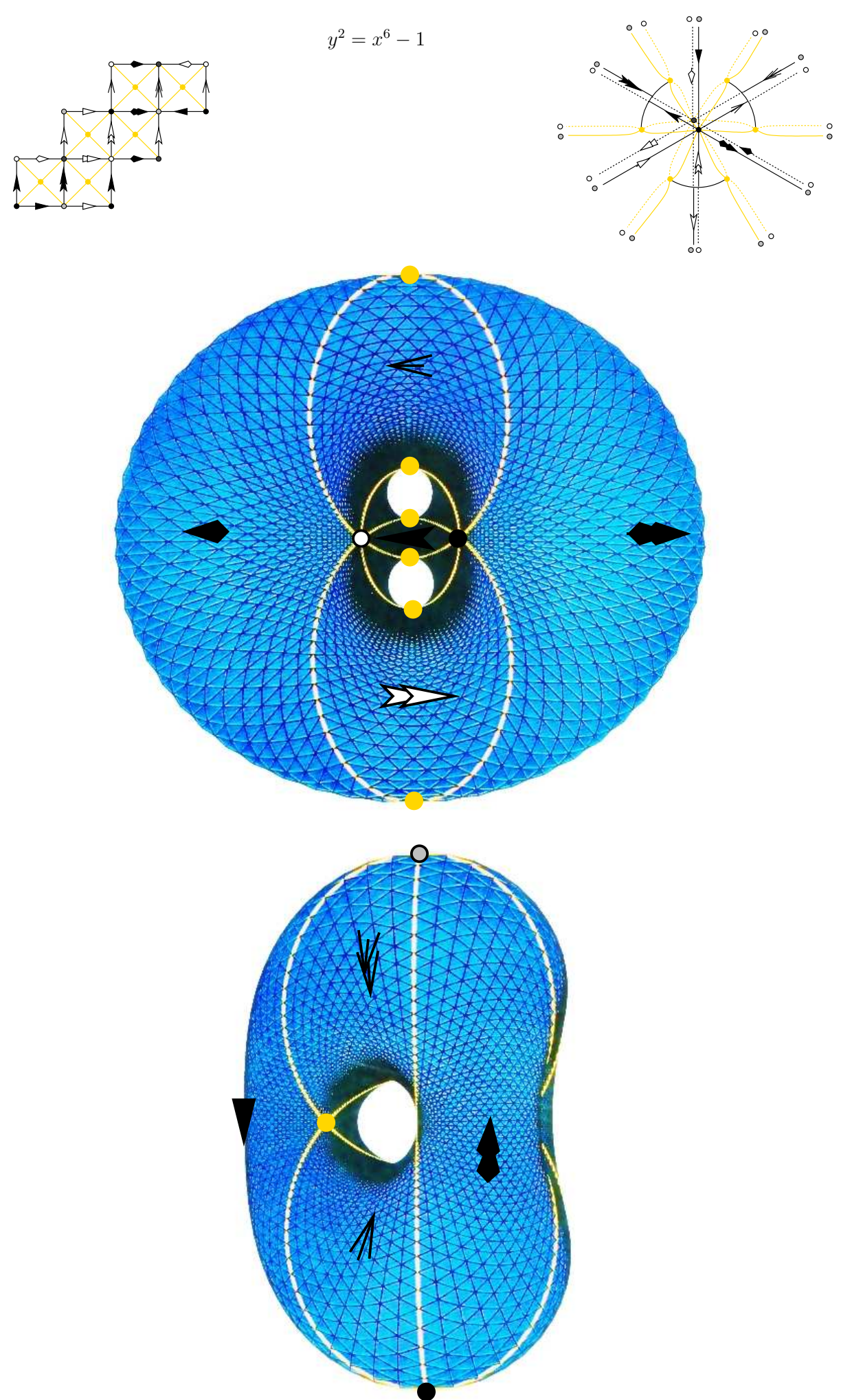

Fig. 6. The Lawson surface is conformally equivalent to a surface made of squares. 


\section{References}

[Abr87] Abresch, U.: Constant mean curvature tori in terms of elliptic functions. J. Reine Angew. Math, 374, 169-192 (1987)

[BBM85] Babich, M. V., Bobenko, A. I., Matveev, V. B.: Solution of nonlinear equations, integrable by the inverse problem method, in Jacobi thetafunctions and the symmetry of algebraic curves. Izv. Akad. Nauk SSSR Ser. Mat., 49(3), 511-529 (1985)

[BCGB08] Ben-Chen, M., Gotsman, C., Bunin, G.: Conformal Flattening by curvature prescription and metric scaling. In Computer Graphics Forum, 27(2). Proc. Eurographics (2008)

[BMS05] Bobenko, A. I, Mercat, C., Suris, Y. B.: Linear and nonlinear theories of discrete analytic functions. Integrable structure and isomonodromic Green's function. J. Reine Angew. Math., 583, 117-161 (2005)

[Bob91] Bobenko, A. I.: All constant mean curvature tori in $\mathbf{R}^{3}, S^{3}, H^{3}$ in terms of theta-functions. Math. Ann., 290(2), 209-245 (1991)

[BS01] Buser, P., Silhol, R.: Geodesics, periods, and equations of real hyperelliptic curves. Duke Math. J., 108(2), 211-250 (2001)

[BS07] Bobenko, A. I., Springborn, B. A.: A discrete Laplace-Beltrami operator for simplicial surfaces. Discrete Comput. Geom., 38(4), 740-756 (2007)

[CSM02] Costa-Santos, R., McCoy, B. M.: Dimers and the critical Ising model on lattices of genus $>1$. Nuclear Phys. B, 623(3), 439-473 (2002)

[CSM03] Costa-Santos, R., McCoy, B. M.: Finite size corrections for the Ising model on higher genus triangular lattices. J. Statist. Phys., 112(5-6), 889-920 (2003)

$\left[\mathrm{DHB}^{+}\right.$04] Deconinck, B., Heil, M., Bobenko, A. I., van Hoeij, M., Schmies, M.: Computing Riemann theta functions. Math. Comp., 73(247), 1417-1442 (2004)

[DKT08] Desbrun, M., Kanso, E., Tong, Y.: Discrete differential forms for computational modeling. In Bobenko, A. I., Schröder, P., Sullivan, J. M., Ziegler, G. M. (eds): Discrete Differential Geometry, Oberwolfach Seminars 38, 287-323. Birkhäuser (2008)

[DMA02] Desbrun, M., Meyer, M., Alliez, P.: Intrinsic Parameterizations of surface meshes. Computer Graphics Forum, 21, 209-218. Proc. Eurographics (2002)

[Duf68] Duffin, R. J.: Potential theory on a rhombic lattice. J. Combinatorial Theory, 5, 258-272 (1968)

[DN03] Dynnikov, I. A., Novikov, S. P.: Geometry of the triangle equation on two-manifolds. Mosc. Math. J., 3(2), 419-438 (2003)

[Fer44] Ferrand, J.: Fonctions préharmoniques et fonctions préholomorphes. Bull. Sci. Math. (2), 68, 152-180 (1944)

[FSSB07] Fisher, M., Springborn, B., Schröder, P., Bobenko, A. I.: An algorithm for the construction of intrinsic Delaunay triangulations with applications to digital geometry processing. Computing, 81(2-3), 199-213 (2007)

[GN07] Grinevich, P. G., Novikov, R. G.: The Cauchy kernel for the NovikovDynnikov DN-discrete complex analysis in triangular lattices. Russ. Math. Surv. 62(4), 799-801 (2007) 
[GBP96] Grosse-Brauckmann, K., Polthier, K.: Numerical examples of compact surfaces of constant mean curvature. In Chow, B., Gulliver, R., Levy, S., Sullivan, J. (eds.) Elliptic and parabolic methods in geometry, 23-46, A K Peters, Wellesley (1996)

[Hei96] Heil, M.: Numerical tools for the study of finite gap solutions of integrable systems. PhD thesis, TU Berlin (1995)

[JWYG04] Jin, M., Wang, Y., Yau, S.-T., Gu, X.: Optimal global conformal surface parameterization. In VIS '04: Proceedings of the conference on Visualization '04 (2004)

[KSS06] Kharevych, L., Springborn, B., Schröder, P.: Discrete conformal mappings via circle patterns. ACM Trans. Graph., 25(2), 412-438 (2006)

[Kis08] Kiselman, C.: Functions on discrete sets holomorphic in the sense of Ferrand, or monodiffric functions of the second kind. Sci. China, Ser. A, 51(4), 604-619 (2008)

[Law70] Blaine Lawson, Jr., H.: Complete minimal surfaces in $S^{3}$. Ann. of Math. (2), 92, 335-374 (1970)

[Mer01] Mercat, C.: Discrete Riemann surfaces and the Ising model. Comm. Math. Phys., 218(1), 177-216 (2001)

[Mer04] Mercat, C.: Exponentials form a basis of discrete holomorphic functions on a compact. Bull. Soc. Math. France, 132(2), 305-326 (2004)

[Mer07] Mercat, C.: Discrete Riemann surfaces. In Papadopoulos, A. (ed) Handbook of Teichmüller Theory, vol. I, IRMA Lect. Math. Theor. Phys., 11, 541-575. Eur. Math. Soc., Zürich (2007)

[PP93] Pinkall, U., Polthier, K.: Computing discrete minimal surfaces and their conjugates. Experiment. Math., 2(1), 15-36 (1993)

[RGA97] Rodríguez, R. E., González-Aguilera, V.: Fermat's quartic curve, Klein's curve and the tetrahedron. In Quine, J. R., Sarnak, P. (ed) Extremal Riemann surfaces. Contemporary Mathematics, 201 AMS (1997)

[Sil01] Silhol, R.: Period matrices and the Schottky problem. In Topics on Riemann surfaces and Fuchsian groups (Madrid, 1998), volume 287 of London Math. Soc. Lecture Note Ser., 287, 155-163. Cambridge Univ. Press, Cambridge (2001)

[Sil06] Silhol, R.: Genus 2 translation surfaces with an order 4 automorphism. In The geometry of Riemann surfaces and abelian varieties. Contemp. Math., 397, 207-213. Amer. Math. Soc., Providence, RI (2006)

[SSP08] Springborn, B., Schröder, P., Pinkall, U.: Conformal equivalence of triangle meshes. SIGGRAPH '08: ACM SIGGRAPH 2008 papers, 1-11. ACM, New York, NY, USA (2008)

[TACSD06] Tong, Y., Alliez, P., Cohen-Steiner, D., Desbrun, M.: Designing quadrangulations with discrete harmonic forms. In Sheffer, A., Polthier, K. (eds) Symposium on Geometry Processing, 201-210, Cagliari, Sardinia, Italy (2006) Eurographics Association.

[Wal89] Walter, R.: Constant mean curvature tori with spherical curvature lines in non-Euclidean geometry. Manuscripta Math., 63(3), 343-363 (1989)

[War06] Wardetzky, M.: Discrete Differential Operators on Polyhedral Surfaces - Convergence and Approximation. PhD thesis, FU Berlin (2006)

[Wen86] Wente, H. C.: Counterexample to a conjecture of H. Hopf. Pacific J. Math., 121(1), 193-243 (1986) 